\title{
More than just a screen to liberate from mechanical ventilation: treat to keep extubated?
}

\author{
Jie Li ${ }^{1}$, J. Brady Scott ${ }^{1}$, Jun Duan ${ }^{2}$, Kai Liu ${ }^{3}$, James B. Fink ${ }^{1,4}$ \\ ${ }^{1}$ Department of Cardiopulmonary Sciences, Division of Respiratory Care, Rush University Medical Center, Chicago, IL, USA; ${ }^{2}$ Department of \\ Respiratory and Critical Care Medicine, the First Affiliated Hospital of Chongqing Medical University, Chongqing 400016, China; ${ }^{3}$ Department of \\ Critical Care Medicine, Shanghai Zhongshan Hospital, Fu Dan University, Shanghai 200032, China; ${ }^{4}$ Aerogen Pharma Corp, San Mateo, CA, USA \\ Correspondence to: James B. Fink. Department of Cardiopulmonary Sciences, Division of Respiratory Care, Rush University Medical Center, Aerogen \\ Pharma Corp., 1525 Seneca Lane: San Mateo, CA 94402, USA. Email: fink.jim@gmail.com. \\ Provenance: This is an invited article commissioned by the Section Editor Dr. Guo-Wei Tu (Department of Critical Care Medicine, Zhongshan \\ Hospital, Fudan University, Shanghai, China). \\ Comment on: Subirà C, Hernández G, Vázquez A, et al. Effect of pressure support vs T-piece ventilation strategies during spontaneous breathing trials \\ on successful extubation among patients receiving mechanical ventilation: A randomized clinical trial. JAMA 2019;321:2175-82.
}

Submitted Sep 10, 2019. Accepted for publication Sep 18, 2019.

doi: 10.21037/atm.2019.09.100

View this article at: http://dx.doi.org/10.21037/atm.2019.09.100

As a life-support modality, mechanical ventilation (MV) has been utilized to support ventilation and oxygenation for patients with respiratory compromise for over five decades. While MV saves lives, it is associated with considerable and relevant clinical complications such as ventilatorinduced lung injury (VILI), ventilator-associated events (VAE), and ventilator-induced diaphragmatic dysfunction (VIDD) $(1,2)$. Thus, efforts to liberate patients from MV as early as possible are common and play a key role in clinical outcomes $(2,3)$. The task to liberate a patient from $M V$ is complicated, as premature extubation has been reported to be harmful. In fact, the mortality for patients who failed initial extubation and required subsequent reintubation has been reported to be 4-9 times higher than those successfully extubated (4-7). In contrast, delayed extubation unnecessarily extends the duration of $\mathrm{MV}$, increasing the likelihood of MV complications. Therefore, identifying the optimal time to liberate patients from MV is crucial.

Spontaneous breathing trials (SBT) were broadly introduced by Esteban et al. in 1995 as a tool to assess patients' readiness to be weaned from MV. Their study demonstrated that daily SBTs were superior to other traditional weaning strategies used at that time (8). Since then, SBTs have become the standard method used during the process of MV liberation (9). While common in clinical practice, there is no consensus on the optimal application of SBTs. In a recent study completed in the United Kingdom, 364 patients were enrolled from 41 different facilities. In that study, 51.1\% of SBTs were performed with continuous positive airway pressure (CPAP), $32.7 \%$ were pressure support ventilation (PSV), and $11.3 \%$ were placed on a T-piece (10). In a study by Jaber et al. with 1,453 patients enrolled from 26 centers in France, SBTs were performed on 1,160 patients, of whom $43.8 \%$ were placed on a T-piece, $38.7 \%$ used PSV with positive end expiratory pressure (PEEP) and $15.8 \%$ used PSV without PEEP (7). Another investigation of 630 patients in 8 North American ICUs showed that the utilization of ventilator with PSV mode was the prevalent modality to implement SBT. Interestingly, the MV settings varied by site as 6 out of $8(75 \%)$ centers preferred PEEP at $5 \mathrm{cmH}_{2} \mathrm{O}$, while the other two centers preferred PEEP at $0 \mathrm{cmH}_{2} \mathrm{O}$. Also, 6 of $8(75 \%)$ of those centers preferred a PS setting of 5-6 $\mathrm{cmH}_{2} \mathrm{O}$ while two centers preferred PS at $0 \mathrm{cmH}_{2} \mathrm{O}(11)$.

Efforts to understand the best method of SBT have been made over time. Esteban et al. compared PSV with T-piece in a randomized controlled trial (RCT) and found that SBTs done with PSV had higher success rates than T-piece ( $86 \%$ vs. $78 \%, \mathrm{P}=0.03$ ) (4). In a physiologic meta-analysis by Sklar et al., work of breathing during SBT with T-piece trials or CPAP at $0 \mathrm{cmH}_{2} \mathrm{O}$ was evaluated and found to 


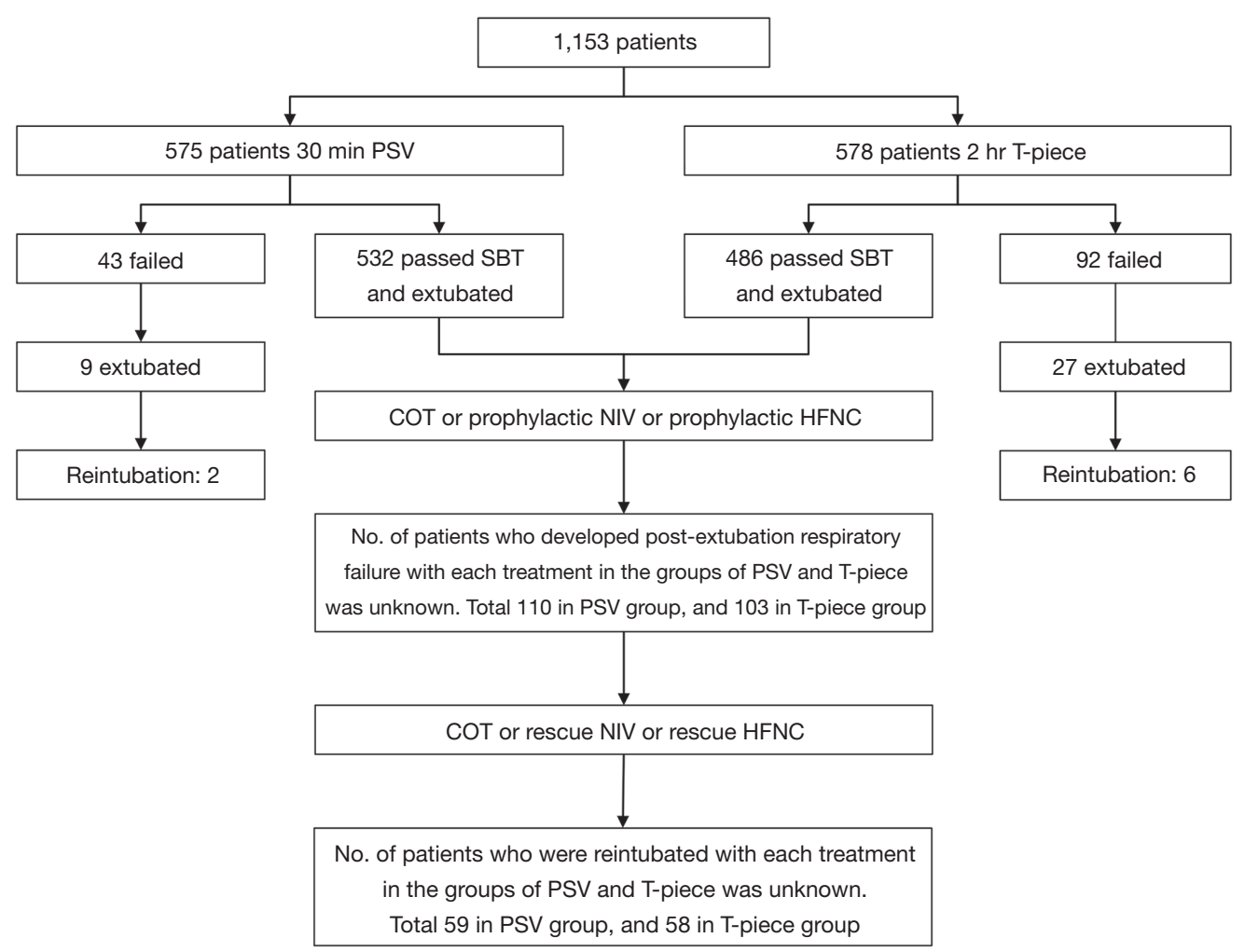

Figure 1 In the Subirà et al.'s study, among the 575 patients who were assigned to receive 30 min PSV SBT, 532 patients passed SBT and were extubated. The remaining 43 patients failed SBT, however 9 were still extubated. In those 541 extubated patients, 51 of them received prophylactic NIV and 91 received prophylactic HFNC. The exact number of patients who received prophylactic NIV and HFNC were unknown in the 532 patients who passed the SBT and were extubated. One hundred and ten in 532 patients developed post-extubation respiratory failure, but the number of patients in the groups of COT, prophylactic NIV and HFNC were unreported. For those 110 patients, some might have changed respiratory support devices, such as from COT to HFNC or NIV, or from HFNC to NIV, as a "rescue therapy", 59 patients ended with reintubation. The number of patients in those rescue treatment groups were unknown. Similar situation also exists in the group of 2-hour T-piece SBT. NIV, noninvasive ventilation; HFNC, high-flow nasal cannula; COT, conventional oxygen therapy.

be similar to work of breathing after extubation. It was also noted, unsurprisingly, that PSV reduced respiratory workload (12). This report might help explain the results from a meta-analysis by Burns et al. assessing outcomes with different SBT modalities (PSV vs. T-piece). Their study demonstrated that patients were more likely to pass a SBT when PSV was utilized (13).

Duration of SBT has also been investigated. In a recent study completed in 17 Spanish ICUs with 470 patients enrolled, 91\% received SBT via T-piece. Approximately one third of the group received SBT via T-piece for 30, 60, and 120 minutes, respectively (14). Liang et al. reported incorporating a 120 -minute SBT with PSV where 352 patients successfully completed 30 minutes, however at 120 minutes, 41 (11.6\%) of those 352 failed the SBT (15).
This suggests that patients are more likely to fail SBT when the length of the trial is longer; for example, 120 minutes instead of only 30 minutes. Despite progression in the understanding of SBTs over time, some debate still exists regarding method and duration.

In 2019, Subirà et al. published the results of their randomized clinical trial in JAMA that evaluated two different SBT strategies, PSV for 30 minutes and T-piece for 120 minutes. The authors provided some insight on whether a less demanding approach to SBT (30-minute PSV) or a more demanding approach (120-minute T-piece) was better. Using successful extubation (free from MV 72 hours after first SBT) as the outcome, they reported that the PSV approach was advantageous in terms of higher rates of successful extubation. While this study provides 
clinicians with more clarity in terms of the application of SBT, there are some important items to consider. First, in the 30-minute PSV group, 142 patients $(24.7 \%)$ received prophylactic noninvasive ventilation (NIV) or high-flow nasal cannula (HFNC) immediately after extubation, which was determined by physicians prior to extubation. In contrast, only 108 patients (18.7\%) received prophylactic NIV or HFNC after extubation in the 2-hour T-piece group (16). The $\mathrm{P}$ value of 0.01 between groups indicates that the baseline data is not comparable. Both NIV and HFNC have been shown to reduce reintubation in patients who successfully completed a SBT (17-20). Therefore, the higher proportion of prophylactic intervention with NIV or HFNC in the 30-minute PSV group in Subirà et al.'s study may have reduced the need for reintubation, resulting in the reported similar reintubation rate compared with $2 \mathrm{~h}$ T-piece group. This raises concern that the 30-minute PSV SBT may not be superior to a 2-hour T-piece if prophylactic interventions of NIV or HFNC were not used.

Another concern is that the use of NIV or HFNC to treat post-extubation respiratory failure, namely "rescue therapy", was not predefined in Subirà et al.'s study. Postextubation respiratory failure occurred in 110 patients $(20.7 \%)$ in the PSV group and in 103 patients $(21.2 \%)$ in the T-piece group. Patients who received HFNC and NIV to treat post-extubation respiratory failure as rescue therapy had greater success avoiding reintubation $(57.4 \%$ and $60.4 \%$, respectively), compared to patients receiving conventional oxygen therapy $(19.7 \% ; \mathrm{P}<0.05)(16)$. It is difficult to distinguish how many patients received rescue therapy by NIV or HFNC and how the treatment (NIV or HFNC) was determined. These data would be helpful to understand whether 30 -min PSV SBT is superior to 2-h T-piece weaning trial (Figure 1). Nonetheless, it appears prophylactic and rescue use of NIV and HFNC vs. conventional $\mathrm{O}_{2}$ may have a role in further reducing reintubation of "simple" weaning patients $(21,22)$.

It needs to be emphasized that SBTs only assesses respiratory function, regardless of the modalities used during SBT. It does not assess upper airway patency, secretion characteristics, and patient's ability to protect airway. As such, Epstein et al. classified extubation failure as airway and non-airway failure (23), in which the incidence of extubation failure due to airway failure was $44-74 \%(7,14,16,18)$. The inability to manage secretions is one of the main reasons for reintubation, suggesting that adequate secretion clearance plays an important role in avoiding reintubation. Wang et al. evaluated secretion clearance methods and their impact on extubation success with a chest physiotherapy treatment protocol for patients whose average MV duration was 7 days. The protocol included chest wall mobilization, secretion removal, cough function training, early mobility, manual hyperinflation and inspiratory muscle training. Compared to a historical control group of patients with conventional care, the patients who received the physiotherapy during and after extubation had lower reintubation rates ( $8 \%$ vs. $16 \%$, $\mathrm{P}=0.01$ ), after successfully passing SBT (24). These findings suggest a role for early assessment of airway patency, secretion clearance, and airway protection abilities with implementation of physiotherapy prior to any extubation.

In conclusion, the key for successful extubation extends beyond screening patients for extubation readiness, to treating patients who might benefit from continued support post-extubation. More work is needed to understand when and which prophylactic measures such as NIV, HFNC and aggressive physiotherapy can reduce risk of reintubation.

\section{Acknowledgments}

None.

\section{Footnote}

Conflicts of Interest: Dr. Fink is Chief Science Officer for Aerogen Pharma Corp and discloses relationships with Dance Biopharm. Mr. Scott discloses relationship with Ventec Life Systems. The other authors have no conflicts of interest to declare.

Ethical Statement: The authors are accountable for all aspects of the work in ensuring that questions related to the accuracy or integrity of any part of the work are appropriately investigated and resolved.

\section{References}

1. Wolkewitz M, Palomar-Martinez M, Alvarez-Lerma F, et al. Analyzing the impact of duration of ventilation, hospitalization, and ventilation episodes on the risk of pneumonia. Infect Control Hosp Epidemiol 2019;40:301-6.

2. Petrof BJ. Diaphragm weakness in the critically ill: Basic mechanisms reveal therapeutic opportunities. Chest 2018;154:1395-403.

3. Kallet RH. Ventilator bundles in transition: From 
prevention of ventilator-associated pneumonia to prevention of ventilator-associated events. Respir Care 2019;64:994-1006.

4. Esteban A, Alía I, Gordo F, et al. Extubation outcome after spontaneous breathing trials with T-tube or pressure support ventilation. Am J Respir Crit Care Med 1997;156:459-65.

5. Esteban A, Alía I, Tobin MJ, et al. Effect of spontaneous breathing trial duration on outcome of attempts to discontinue mechanical ventilation. Am J Respir Crit Care Med 1999;159:512-8.

6. Perren A, Domenighetti G, Mauri S, et al. Protocoldirected weaning from mechanical ventilation: clinical outcome in patients randomized for a 30-min or 120-min trial with pressure support ventilation. Intensive Care Med 2002;28:1058-63.

7. Jaber S, Quintard H, Cinotti R, et al. Risk factors and outcomes for airway failure versus non-airway failure in the intensive care unit: a multicenter observational study of 1514 extubation procedures. Crit Care 2018;22:236.

8. Esteban A, Frutos F, Tobin MJ, et al. A comparison of four methods of weaning patients from mechanical ventilation. N Engl J Med 1995;332:345-50.

9. Schmidt GA, Girard TD, Kress JP, et al. Liberation from mechanical ventilation in critically ill adults: executive summary of an official American College of Chest Physicians/American Thoracic Society clinical practice guideline. Chest 2017;151:160-5.

10. Perkins GD, Mistry D, Gates S, et al. Effect of protocolized weaning with early extubation to noninvasive ventilation vs invasive weaning on time to liberation from mechanical ventilation among patients with respiratory failure: The breathe randomized clinical trial. JAMA 2018;320:1881-8.

11. Godard S, Herry C, Westergaard P, et al. Practice variation in spontaneous breathing trial performance and reporting. Can Respir J 2016;2016:9848942.

12. Sklar MC, Burns K, Rittayamai N, et al. Effort to breathe with various spontaneous breathing trial techniques. A physiologic meta-analysis. Am J Respir Crit Care Med 2017;195:1477-85.

13. Burns KEA, Soliman I, Adhikari NK, et al. Trials directly comparing alternative spontaneous breathing trial techniques: a systematic review and meta-analysis. Crit Care 2017;21:127.

14. Fernandez MM, González-Castro A, Magret M, et al. Reconnection to mechanical ventilation for $1 \mathrm{~h}$ after a successful spontaneous breathing trial reduces reintubation in critically ill patients: a multicenter randomized controlled trial. Intensive Care Med 2017;43:1660-7.

15. Liang G, Liu T, Zeng Y, et al. Characteristics of subjects who failed a 120-minute spontaneous breathing trial. Respir Care 2018;63:388-94.

16. Subirà $\mathrm{C}$, Hernández $\mathrm{G}$, Vázquez $\mathrm{A}$, et al. Effect of pressure support vs $\mathrm{T}$-piece ventilation strategies during spontaneous breathing trials on successful extubation among patients receiving mechanical ventilation: A randomized clinical trial. JAMA 2019;321:2175-82.

17. Zhu Y, Yin H, Zhang R, et al. High-flow nasal cannula oxygen therapy versus conventional oxygen therapy in patients after planned extubation: a systematic review and meta-analysis. Crit Care 2019;23:180.

18. Hernández G, Vaquero C, Colinas L, et al. Effect of postextubation high-flow nasal cannula vs noninvasive ventilation on reintubation and postextubation respiratory failure in high-risk patients: A randomized clinical trial. JAMA 2016;316:1565-74.

19. Glossop AJ, Shephard N, Bryden DC, et al. Noninvasive ventilation for weaning, avoiding reintubation after extubation and in the postoperative period: a metaanalysis. Br J Anaesth 2012;109:305-14.

20. Lin C, Yu H, Fan H, et al. The efficacy of noninvasive ventilation in managing postextubation respiratory failure: a meta-analysis. Heart Lung 2014;43:99-104.

21. Pellegrini JA, Moraes RB, Maccari JG, et al. Spontaneous breathing trials with T-piece or pressure support ventilation. Respir Care 2016;61:1693-703.

22. Funk GC, Anders S, Breyer MK, et al. Incidence and outcome of weaning from mechanical ventilation according to new categories. Eur Respir J 2010;35:88-94.

23. Epstein SK, Ciubotaru RL. Independent effects of etiology of failure and time to reintubation on outcome for patients failing extubation. Am J Respir Crit Care Med 1998;158:489-93.

24. Wang TH, Wu CP, Wang LY. Chest physiotherapy with early mobilization may improve extubation outcome in critically ill patients in the intensive care units. Clin Respir J 2018;12:2613-21.

Cite this article as: Li J, Scott JB, Duan J, Liu K, Fink JB. More than just a screen to liberate from mechanical ventilation: treat to keep extubated? Ann Transl Med 2019;7(Suppl 8):S338. doi: 10.21037/atm.2019.09.100 